PROCEEDINGS OF THE

AMERICAN MATHEMATICAL SOCIETY

Volume 138, Number 3, March 2010, Pages 1131-1142

S 0002-9939(09)10128-4

Article electronically published on October 26, 2009

\title{
LANTERN RELATIONS AND RATIONAL BLOWDOWNS
}

\author{
HISAAKI ENDO AND YUSUF Z. GURTAS
}

(Communicated by Daniel Ruberman)

\begin{abstract}
We discuss a connection between the lantern relation in mapping class groups and the rational blowing down process for 4-manifolds. More precisely, if we change a positive relator in Dehn twist generators of the mapping class group by using a lantern relation, the corresponding Lefschetz fibration changes into its rational blowdown along a copy of the configuration $C_{2}$. We exhibit examples of such rational blowdowns of Lefschetz fibrations whose blowup is homeomorphic but not diffeomorphic to the original fibration.
\end{abstract}

\section{INTRODUCTION}

Lefschetz fibrations relate the topology of symplectic 4-manifolds to the combinatorics on positive relators in Dehn twist generators of mapping class groups. Fuller introduced a substitution technique for constructing positive relators to obtain an example of non-holomorphic Lefschetz fibrations of genus three [15], 14]. Many constructions of Lefschetz fibrations as positive relators can be interpreted as generalizations of his construction (cf. [5]), while what such substitutions mean geometrically has been less investigated.

In this paper we study a particular substitution, the lantern substitution (or the $L^{ \pm 1}$-substitution for short), for positive relators of mapping class groups. The corresponding surgical operation on Lefschetz fibrations turns out to be the rational blowing down process, which was discovered by Fintushel and Stern [6], along with a copy of the configuration $C_{2}$ (i.e., a -4-framed unknot in Kirby diagrams). Applying a theorem of Usher [19, we give examples of such rational blowdowns of Lefschetz fibrations whose blowup is homeomorphic but not diffeomorphic to the original fibration.

In Section 2 we review the lantern relation in mapping class groups and define the lantern substitution for positive relators. We discuss a relation between lantern relations and rational blowdowns in Section 3 and state the main theorem in Section 4. We then exhibit some examples in Section 5 and end by observing other relations in Section 6.

Received by the editors November 21, 2008, and, in revised form, July 20, 2009.

2010 Mathematics Subject Classification. Primary 57R17; Secondary 57N13, 20F38.

Key words and phrases. 4-manifold, mapping class group, symplectic topology, Lefschetz fibration, lantern relation, rational blowdown.

The first author is partially supported by Grant-in-Aid for Scientific Research (No. 21540079), Japan Society for the Promotion of Science. 
The authors are grateful to Y. Sato for useful comments on Problem 5.4, to K. Yasui for helpful comments on the rational blowing-down process, and to N. Monden for drawing beautiful Kirby diagrams in Figure 1 and Figure 2.

\section{LANTERN RELATIONS AND SUBSTITUTIONS}

Let $\Sigma_{g}$ be a closed oriented surface of genus $g(\geq 2)$ and $\mathcal{M}_{g}$ the mapping class group of $\Sigma_{g}$. We denote by $\mathcal{F}$ the free group generated by all isotopy classes $\mathcal{S}$ of simple closed curves on $\Sigma_{g}$. There is a natural epimorphism $\varpi: \mathcal{F} \rightarrow \mathcal{M}_{g}$ which sends (the isotopy class of) a simple closed curve $a$ on $\Sigma_{g}$ to the righthanded Dehn twist $t_{a}$ along $a$. We set $\mathcal{R}:=$ Ker $\varpi$ and call each element of $\mathcal{R}$ a relator in the generators $\mathcal{S}$ of $\mathcal{M}_{g}$. A word in the generators $\mathcal{S}$ is called positive if it includes no negative exponents. We put ${ }_{W}(c):=t_{a_{r}}^{\varepsilon_{r}} \cdots t_{a_{1}}^{\varepsilon_{1}}(c) \in \mathcal{S}$ for $c \in \mathcal{S}$ and $W=a_{r}^{\varepsilon_{r}} \cdots a_{1}^{\varepsilon_{1}} \in \mathcal{F}\left(a_{1}, \ldots, a_{r} \in \mathcal{S}, \varepsilon_{1}, \ldots, \varepsilon_{r} \in\{ \pm 1\}\right)$, and put ${ }_{W} V:={ }_{W}\left(c_{1}\right) \cdots_{W}\left(c_{s}\right) \in \mathcal{F}$ for $V=c_{1} \cdots c_{s} \in \mathcal{F}\left(c_{1}, \ldots, c_{s} \in \mathcal{S}\right)$.

We begin with a precise definition of the lantern relation [2], [9].

Definition 2.1. Let $a$ and $b$ be simple closed curves on $\Sigma_{g}$ with geometric intersection number 2 and algebraic intersection number 0 . We orient $a$ and $b$ locally on a neighborhood of each intersection point $p \in a \cap b$ such that the intersection number $(a \cdot b)_{p}$ at $p$ is +1 . Resolving all intersection points according to the local orientations, we obtain a new simple closed curve $c$. A regular neighborhood of $a \cup b$, which can be chosen to include $c$, is a genus 0 subsurface $\Sigma$ of $\Sigma_{g}$ with four boundary components. We denote simple closed curves parallel to four boundary components of $\Sigma$ by $d_{1}, d_{2}, d_{3}$, and $d_{4}$. The relation

$$
t_{d_{1}} t_{d_{2}} t_{d_{3}} t_{d_{4}}=t_{a} t_{b} t_{c}
$$

is called the lantern relation. We put $L:=L(a, b)=a b c d_{4}^{-1} d_{3}^{-1} d_{2}^{-1} d_{1}^{-1} \in \mathcal{R}$.

Let $\varrho \in \mathcal{R}(\varrho \neq 1)$ be a positive relator of $\mathcal{M}_{g}$. Let $a, b, c, d_{1}, d_{2}, d_{3}$, and $d_{4}$ be curves as in Definition 2.1. Suppose that $\varrho$ includes $d_{1} d_{2} d_{3} d_{4}$ as a subword: $\varrho=U \cdot d_{1} d_{2} d_{3} d_{4} \cdot V(U, V \in \mathcal{F})$. Since $\varrho$ and $U \cdot L \cdot U^{-1}$ are both relators of $\mathcal{M}_{g}$, the positive word

$$
\begin{aligned}
& \varrho^{\prime}=U \cdot a b c \cdot V\left(=U \cdot a b c d_{4}^{-1} d_{3}^{-1} d_{2}^{-1} d_{1}^{-1} \cdot d_{1} d_{2} d_{3} d_{4} \cdot V\right. \\
& \left.\quad=U \cdot L \cdot U^{-1} \cdot U \cdot d_{1} d_{2} d_{3} d_{4} \cdot V=U \cdot L \cdot U^{-1} \cdot \varrho\right)
\end{aligned}
$$

is also a relator of $\mathcal{M}_{g}$. The length of the word $\varrho^{\prime}$ is equal to that of $\varrho$ minus one.

Definition 2.2. We say that $\varrho^{\prime}$ is obtained by applying an $L$-substitution to $\varrho$. Conversely, $\varrho$ is said to be obtained by applying an $L^{-1}$-substitution to $\varrho^{\prime}$. We also call these two kinds of operations lantern substitutions (cf. [5]).

We next recall a definition of Lefschetz fibrations (cf. [13], [8]).

Definition 2.3. Let $M$ be a closed oriented smooth 4-manifold. A smooth map $f: M \rightarrow S^{2}$ is called a Lefschetz fibration of genus $g$ if it satisfies the following conditions:

(i) $f$ has finitely many critical values $b_{1}, \ldots, b_{n} \in S^{2}$ and $f$ is a smooth fiber bundle over $S^{2}-\left\{b_{1}, \ldots, b_{n}\right\}$ with fiber $\Sigma_{g}$;

(ii) for each $i(i=1, \ldots, n)$, there exists a unique critical point $p_{i}$ in the singular fiber $f^{-1}\left(b_{i}\right)$ such that $f$ is locally written as $f\left(z_{1}, z_{2}\right)=z_{1}^{2}+z_{2}^{2}$ with respect to some 
local complex coordinates around $p_{i}$ and $b_{i}$ which are compatible with orientations of $M$ and $S^{2}$;

(iii) no fiber contains a -1 -sphere.

Remark 2.4. A more general definition can be found in Chapter 8 of 8 . We treat also Lefschetz fibrations with boundary in the proof of Theorem 3.1

Suppose that $g \geq 2$. According to theorems of Kas and Matsumoto, there exists a one-to-one correspondence between the isomorphism classes of Lefschetz fibrations and the equivalence classes of positive relators modulo simultaneous conjugations

$$
c_{1} \cdots c_{n} \sim{ }_{W}\left(c_{1}\right) \cdots_{W}\left(c_{n}\right),
$$

and elementary transformations

$$
\begin{gathered}
c_{1} \cdots c_{i} \cdot c_{i+1} \cdots c_{n} \sim c_{1} \cdots c_{i+1} \cdot{ }_{c_{i+1}^{-1}}\left(c_{i}\right) \cdots c_{n}, \\
c_{1} \cdots c_{i} \cdot c_{i+1} \cdots c_{n} \sim c_{1} \cdots c_{i}\left(c_{i+1}\right) \cdot c_{i} \cdots c_{n},
\end{gathered}
$$

where $c_{1} \cdots c_{n} \in \mathcal{R}$ is a positive relator in the generator $\mathcal{S}$ and $W \in \mathcal{F}$. This correspondence is described by using the holonomy (or monodromy) homomorphism induced by the classifying map of $f$ restricted on $S^{2}-\left\{b_{1}, \ldots, b_{n}\right\}$ (cf. [8, [13, and [3]). We denote (the isomorphism class of) a Lefschetz fibration associated to a positive relator $\varrho \in \mathcal{R}$ by $M_{\varrho} \rightarrow S^{2}$.

Let $\varrho, \varrho^{\prime} \in \mathcal{R}$ be positive relators of $\mathcal{M}_{g}$ and let $M_{\varrho}, M_{\varrho^{\prime}}$ be the corresponding Lefschetz fibrations over $S^{2}$, respectively. Suppose that the relator $\varrho^{\prime}$ is obtained by applying an $L$-substitution to the relator $\varrho$. The Euler characteristic and the signature of a Lefschetz fibration $M_{\varrho^{\prime}} \rightarrow S^{2}$ with monodromy $\varrho^{\prime}$ are computed as follows:

$$
e\left(M_{\varrho^{\prime}}\right)=e\left(M_{\varrho}\right)-1, \quad \sigma\left(M_{\varrho^{\prime}}\right)=\sigma\left(M_{\varrho}\right)+1
$$

([5], Theorem 4.3 and Proposition 3.12). We investigate relations between $M_{\varrho}$ and $M_{\varrho^{\prime}}$ and several properties of them in the subsequent sections.

\section{RATIONAL BLOWDOWNS VIA LANTERN RELATIONS}

Let $\varrho, \varrho^{\prime} \in \mathcal{R}$ be positive relators of $\mathcal{M}_{g}$ and let $M_{\varrho}, M_{\varrho^{\prime}}$ be the corresponding Lefschetz fibrations over $S^{2}$, respectively.

Theorem 3.1. If $\varrho^{\prime}$ is obtained by applying an L-substitution to $\varrho$, then the 4manifold $M_{\varrho^{\prime}}$ is a rational blowdown of $M_{\varrho}$ along a configuration $C_{2} \subset M_{\varrho}$.

Proof. We take a subsurface $\Sigma$ of $\Sigma_{g}$ and curves $a, b, c, d_{1}, d_{2}, d_{3}$, and $d_{4}$ on $\Sigma$ as in Definition 2.1. Let $N, N^{\prime}$ be Lefschetz fibrations over $D^{2}$ with fiber $\Sigma$ corresponding to the positive words $d_{1} d_{2} d_{3} d_{4}, a b c$, respectively.

Drawing a Kirby diagram of $N$, sliding the central -1-framed unknot over the other three -1-framed unknots, and canceling three 1-handle/2-handle pairs, we obtain a -4-framed unknot (Figure 1). Thus $N$ is diffeomorphic to the total space of a $D^{2}$-bundle over $S^{2}$ with Euler number -4, which is denoted by $C_{2}$ in [6] (see also 8], Section 8.5).

Drawing a Kirby diagram of $N^{\prime}$ and sliding and canceling handles as in Figure 2, we obtain a pair of a dotted circle and a +1 -framed unknot with linking number +2 . This means that $N^{\prime}$ is diffeomorphic to a rational 4-ball with boundary $L(4,1)$, which is denoted by $B_{2}$ in $[6$, (see also [8], Section 8.5). 


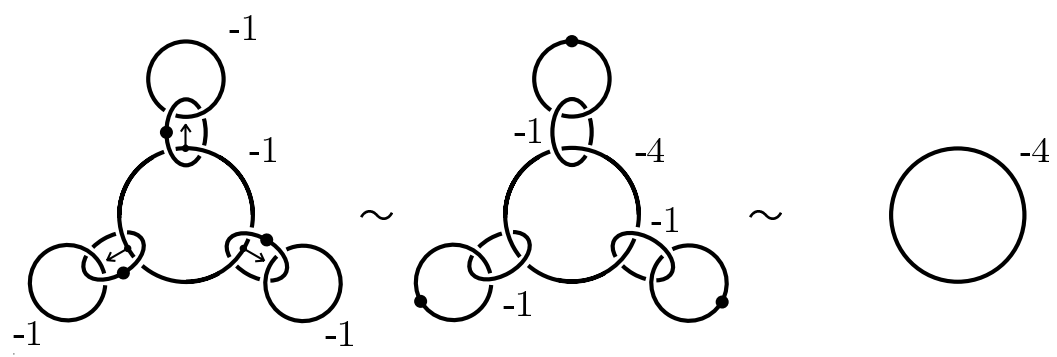

FigURE 1

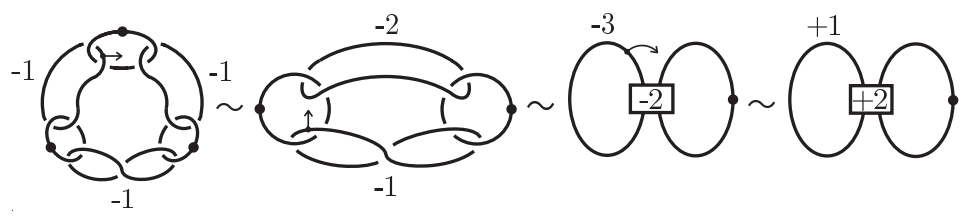

FiguRE 2

From construction, $N$ (resp. $N^{\prime}$ ) can be considered to be a submanifold of $M_{\varrho}$ (resp. $M_{\varrho^{\prime}}$ ). It is also easily seen that $M_{\varrho}-\operatorname{int} N$ and $M_{\varrho^{\prime}}-\operatorname{int} N^{\prime}$ are diffeomorphic to each other. Hence we have

$$
M_{\varrho^{\prime}} \approx N^{\prime} \cup_{\partial N}\left(M_{\varrho}-\operatorname{int} N\right) \approx B_{2} \cup_{L(4,1)}\left(M_{\varrho}-\operatorname{int} C_{2}\right) .
$$

This completes the proof of Theorem 3.1

\section{Smooth structures}

Let $\varrho, \varrho^{\prime} \in \mathcal{R}$ be positive relators of $\mathcal{M}_{g}$ and let $M_{\varrho}, M_{\varrho^{\prime}}$ be the corresponding Lefschetz fibrations over $S^{2}$, respectively. Suppose that $\varrho^{\prime}$ is obtained by applying $k$ times $L$-substitutions $(k \geq 1)$, elementary transformations, and simultaneous conjugations to $\varrho$. Suppose also that $e\left(M_{\varrho}\right)+\sigma\left(M_{\varrho}\right) \geq 2$. We choose a positive relator $\varsigma \in \mathcal{R}(\varsigma \neq 1)$ such that $M_{\varsigma}-\nu F$ is simply-connected and either the word $\varsigma$ includes at least one separating curve as a factor, or $\sigma\left(M_{\varrho}\right)+\sigma\left(M_{\varsigma}\right)$ is not divisible by 16 . Here $\nu F$ is an open fibered neighborhood of a general fiber $F$ of $M_{\varsigma}$. Taking a fiber sum of $M_{\varrho}$ (resp. $M_{\varrho^{\prime}}$ ) with $M_{\varsigma}$, we obtain a new Lefschetz fibration $M_{1}:=M_{\varrho} \#_{F} M_{\varsigma}\left(\right.$ resp. $\left.M_{2}:=M_{\varrho^{\prime}} \#_{F} M_{\varsigma}\right)$ with monodromy $\varrho \cdot{ }_{W} \varsigma\left(\right.$ resp. $\left.\varrho^{\prime} \cdot W^{\prime} \varsigma\right)$ for some $W \in \mathcal{F}$ (resp. $\left.W^{\prime} \in \mathcal{F}\right)$. It is obvious that $\varrho^{\prime} \cdot W^{\prime} \varsigma$ is obtained by applying $k$ times $L$-substitutions, elementary transformations, and simultaneous conjugations to $\varrho \cdot W \varsigma$.

Theorem 4.1. The 4-manifold $M_{1}$ is homeomorphic but not diffeomorphic to a $k$ times blowup $M_{2} \# k \overline{\mathbb{C P}}^{2}$ of $M_{2}$. Moreover, both of these 4-manifolds do not dissolve.

Proof. Let $j: F_{i} \hookrightarrow M_{i}$ be the inclusion map from a general fiber $F_{i}$ into the total space $M_{i}(i=1,2)$. The induced homomorphism $j_{\#}: \pi_{1}\left(F_{i}\right) \rightarrow \pi_{1}\left(M_{i}\right)$ is surjective and the kernel of $j_{\#}$ includes the normal subgroup $N$ of $\pi_{1}\left(M_{i}\right)$ generated by the vanishing cycles of $M_{i}$ (cf. [1], Lemma 3.2). Since $M_{\varsigma}-\nu F$ is simply-connected and $j_{\#}$ is the composition of homomorphisms $\pi_{1}\left(F_{i}\right) \rightarrow \pi_{1}\left(M_{\varsigma}-\nu F\right) \rightarrow \pi_{1}\left(M_{i}\right)$, the group $\pi_{1}\left(M_{i}\right)$ must be trivial $(i=1,2)$. 
$M_{1}$ is a non-spin 4-manifold because either it has a component of a separating singular fiber which represents a homology class of square -1 , or $\sigma\left(M_{1}\right)$ is not divisible by 16. It is easily seen from the observation above that $e\left(M_{2}\right)=e\left(M_{1}\right)-k$ and $\sigma\left(M_{2}\right)=\sigma\left(M_{1}\right)+k$. By virtue of Freedman's classification theorem, both $M_{1}$ and $M_{2} \# k \overline{\mathbb{C P}}^{2}$ are homeomorphic to $\# b_{2}^{+}\left(M_{1}\right) \mathbb{C P}^{2} \# b_{2}^{-}\left(M_{1}\right) \overline{\mathbb{C P}}^{2}$ because they are simply-connected, non-spin, and have the same Euler characteristic and the same signature.

$M_{1}$ is a fiber sum $M_{\varrho} \#_{F} M_{\varsigma}$ of non-trivial Lefschetz fibrations $M_{\varrho}$ and $M_{\varsigma}$. By Gompf's theorem ([8, Theorem 10.2.18), $M_{1}$ admits a symplectic structure with symplectic fibers. It follows from a theorem of Usher [19 that $M_{1}$ is a minimal symplectic 4-manifold. Since $b_{2}^{+}\left(M_{1}\right)=b_{2}^{+}\left(M_{\varrho}\right)-b_{1}\left(M_{\varrho}\right)+b_{2}^{+}\left(M_{\varsigma}\right)+2 g-1>1$, $M_{1}$ does not contain any smooth -1-sphere as a consequence of Seiberg-Witten theory ([17], 18]; cf. 8], Remark 10.2.4(a)). On the other hand, $M_{2} \# k \overline{\mathbb{C P}}^{2}$ has a natural smooth -1 -sphere. Hence $M_{1}$ and $M_{2} \# k \overline{\mathbb{C P}}^{2}$ cannot be diffeomorphic.

Because $M_{1}$ and $M_{2} \# k \overline{\mathbb{C P}}^{2}$ admit symplectic structures and $b_{2}^{+}\left(M_{1}\right)>1$, these manifolds cannot be diffeomorphic to $\# b_{2}^{+}\left(M_{1}\right) \mathbb{C P}^{2} \# b_{2}^{-}\left(M_{1}\right) \overline{\mathbb{C P}}^{2}$ ([16], [10]; cf. [8], Theorem 10.1.14).

Remark 4.2. We do not use any explicit property of rational blowdowns to prove Theorem 4.1. The proof above is rather similar to that of Theorem 4.8 of [3]. It is likely that $M_{\varrho}$ is homeomorphic but not diffeomorphic to $M_{\varrho^{\prime}} \# k \overline{\mathbb{C P}}^{2}$ (without taking fiber sums with $M_{\varsigma}$ ) in a general setting. On the other hand, a certain rational blowdown along $C_{2}$ happens to be diffeomorphic to an honest blowdown of the original 4-manifold: $E(1)_{2}\left(\approx E(1) \approx \mathbb{C P}^{2} \# 9 \overline{\mathbb{C P}}^{2}\right)$ is a rational blowdown of $E(1) \# \overline{\mathbb{C P}}^{2}\left(\approx \mathbb{C P}^{2} \# 10 \overline{\mathbb{C P}}^{2}\right)$ along $C_{2}$ ([6], Proposition 3.2; cf. [8], Theorem 8.5.9 and Theorem 8.3.11).

\section{ExAmples}

We apply theorems in previous sections to explicit examples.

Example 5.1. Suppose that $g \geq 4$. Choose and fix $2 g+1$ simple closed curves $c_{1}, c_{2}, \ldots, c_{2 g+1}$ on $\Sigma_{g}$ such that $c_{i}$ and $c_{i+1}$ intersect transversely at one point for $i=1,2, \ldots, 2 g$ and $c_{i} \cap c_{j}=\emptyset$ if $|i-j|>1$. Let $d_{h, 1}$ and $d_{h, 2}$ be simple closed curves parallel to the boundary of a regular neighborhood of $c_{1} \cup c_{2} \cup \cdots \cup c_{2 h+1}$ for $h=1, \ldots, g-2$. We consider positive words

$$
\begin{aligned}
F_{h-1}^{\text {even }}:= & d_{h-1,1} d_{h-1,2}\left(c_{2 h} c_{2 h-1} \cdots c_{2} c_{1}\right)\left(c_{2 h+1} c_{2 h} \cdots c_{3} c_{2}\right) \\
& \cdots\left(c_{2 g} c_{2 g-1} \cdots c_{2 g-2 h+2} c_{2 g-2 h+1}\right)\left(c_{1} c_{2} \cdots c_{2 g}\right)^{4 g-2 h+2}, \\
\bar{F}_{g-h-1}^{\text {even }}:= & \left(c_{2} c_{3} \cdots c_{2 g+1}\right)^{2 g+2 h+2}\left(c_{2 g-2 h+1} \cdots c_{3} c_{2}\right)\left(c_{2 g-2 h+2} \cdots c_{4} c_{3}\right) \\
& \cdots\left(c_{2 g+1} \cdots c_{2 h+3} c_{2 h+2}\right) d_{h, 1} d_{h, 2}
\end{aligned}
$$

for $h=2, \ldots, g-2$. It was shown in Section 4 of [5] that both $F_{h-1}^{\text {even }}$ and $\bar{F}_{g-h-1}^{\text {even }}$ are positive relators of $\mathcal{M}_{g}$. Since $d_{h-1,1}, d_{h-1,2}, d_{h, 1}$, and $d_{h, 2}$ bound a genus 0 subsurface $\Sigma$ of $\Sigma_{g}$ with four boundary components, we can take simple closed curves $a_{h}, b_{h}$, and $c_{h}$ on $\Sigma$ so that

$$
L=L\left(a_{h}, b_{h}\right):=a_{h} b_{h} c_{h} d_{h-1,1}^{-1} d_{h-1,2}^{-1} d_{h, 1}^{-1} d_{h, 2}^{-1}
$$


is the relator corresponding to a lantern relation. Then we apply an $L$-substitution to $\bar{F}_{g-h-1}^{\text {even }} F_{h-1}^{\text {even }}$ to obtain a positive relator $V_{h}$.

Suppose that $g \geq 2$ and $g$ is even. Let $d$ be the boundary curve of a regular neighborhood of $c_{1} \cup \cdots \cup c_{g}$. It was shown in Section 4 of [3] that the positive word

$$
\begin{aligned}
Q:= & \left(c_{1} c_{2} \cdots c_{2 g+1}\right)^{g+1} \cdot d \cdot W_{1}\left(c_{g+1}\right) \cdot W_{2}\left(c_{g+2}\right) \cdots W_{g+1}\left(c_{2 g+1}\right) \\
& \left(W_{i}:=\left(c_{i+g-1} \cdots c_{i+1} c_{i}\right)^{-1}(i=1, \ldots, g+1)\right)
\end{aligned}
$$

is a positive relator of $\mathcal{M}_{g}$. Suppose that $g \geq 3$ and $g$ is odd. Let $d$ be the boundary curve of a regular neighborhood of $c_{1} \cup \cdots \cup c_{g-1}$. It was shown in Section 4 of [3] that the positive word

$$
\begin{aligned}
Q:= & \left(c_{1} c_{2} \cdots c_{2 g+1}\right)^{g+2} \cdot d \cdot\left(c_{g-1} \cdots c_{2} c_{1}\right)^{2} \cdot W_{1}\left(c_{g}\right) \cdot W_{2}\left(c_{g+1}\right) \cdots W_{g+2}\left(c_{2 g+1}\right) \\
& \left(W_{i}:=\left(c_{i+g-2} \cdots c_{i+1} c_{i}\right)^{-1}(i=1, \ldots, g+2)\right)
\end{aligned}
$$

is a positive relator of $\mathcal{M}_{g}$.

For every $g(\geq 4)$ and $h=2, \ldots, g-2$, we set $\varrho:=\bar{F}_{g-h-1}^{\text {even }} F_{h-1}^{\text {even }}, \varrho^{\prime}:=V_{h}$, and $\varsigma:=Q$. Since $\varrho^{\prime}$ is obtained by applying an $L$-substitution to $\varrho$, it turns out from Theorem 3.1 that $M_{\varrho^{\prime}}$ is a rational blowdown of $M_{\varrho}$ along a copy of $C_{2}$. The Euler characteristic and the signature of $M_{\varrho^{\prime}}$ are equal to $12 g^{2}+6 g+8 g h-8 h^{2}+7$ and $-6 g^{2}-8 g-4 g h+4 h^{2}-3$, respectively. $M_{\varsigma}-\nu F$ is simply-connected and $\varsigma$ includes one separating curve. The Euler characteristic and the signature of $M_{\varsigma}$ are $2 g^{2}+7$ and $-\left(g^{2}+2 g+3\right)$ for even $g$, and $2 g^{2}+4 g+7$ and $-(g+2)^{2}$ for odd $g$, respectively. We set $M_{1}:=M_{\varrho} \#_{F} M_{\varsigma}$ and $M_{2}:=M_{\varrho^{\prime}} \#_{F} M_{\varsigma}$. It follows from Theorem 4.1 that $M_{1}$ is homeomorphic but not diffeomorphic to $M_{2} \# \overline{\mathbb{C P}}^{2}$ and both of these do not dissolve. If we use $Q^{n}(n \geq 2)$ instead of $Q$, we obtain infinitely many pairs of homeomorphic but non-diffeomorphic 4-manifolds for a fixed $g(\geq 4)$.

Example 5.2. The second example is a Lefschetz fibration of genus 3 given by an order-three element in $\mathcal{M}_{3}$ as defined in $\S 3.2$ of [4]. Consider the relations

$$
\left(t_{c_{1}} t_{c_{2}}\right)^{6}=t_{\bar{d}_{1}}=t_{\delta_{1}}, \quad\left(t_{c_{4}} t_{e_{1}} t_{c_{4}} t_{f_{1}}\right)^{3}=t_{d_{2}} t_{\bar{d}_{2}}=t_{\delta_{1}} t_{\delta_{2}}, \quad \text { and } \quad\left(t_{c_{7}} t_{c_{6}}\right)^{6}=t_{d_{3}}=t_{\delta_{2}}
$$

on tori with one and two components, Figure 3, which can also be seen as subsurfaces of a closed surface of genus three, Figure 4. The order-three element on this surface is obtained by writing

$$
\begin{aligned}
& \left(t_{c_{1}} t_{c_{2}}\right)^{6} t_{\delta_{1}}^{-1}\left(t_{c_{4}} t_{e_{1}} t_{c_{4}} t_{f_{1}}\right)^{-3} t_{\delta_{1}} t_{\delta_{2}}\left(t_{c_{7}} t_{c_{6}}\right)^{6} t_{\delta_{2}}^{-1} \\
= & \left(\left(t_{c_{1}} t_{c_{2}}\right)^{2}\left(t_{c_{4}} t_{e_{1}} t_{c_{4}} t_{f_{1}}\right)^{-1}\left(t_{c_{7}} t_{c_{6}}\right)^{2}\right)^{3}=1
\end{aligned}
$$

after gluing together the bounded tori with alternating orientations. We need to eliminate the negative exponent in this expression in order to obtain a positive relator. We do this in a few steps. First, we write $\left(t_{c_{4}} t_{e_{1}} t_{c_{4}} t_{f_{1}}\right)^{3}=t_{\delta_{1}} t_{\delta_{2}}$ as $\left(t_{c_{4}} t_{e_{1}} t_{c_{4}} t_{f_{1}}\right)^{-1}=t_{\delta_{1}}^{-1}\left(t_{c_{4}} t_{e_{1}} t_{c_{4}} t_{f_{1}}\right)^{2} t_{\delta_{2}}^{-1}$. Next, we replace $t_{\delta_{1}}^{-1}$ and $t_{\delta_{2}}^{-1}$ using lantern relations $t_{\delta_{1}} t_{x_{1}} t_{c_{3}}=t_{c_{1}} t_{c_{1}} t_{e_{1}} t_{f_{1}}$ and $t_{\delta_{2}} t_{x_{2}} t_{c_{5}}=t_{e_{1}} t_{f_{1}} t_{c_{7}} t_{c_{7}}$. Solving these relations for $t_{\delta_{1}}^{-1}$ and $t_{\delta_{2}}^{-1}$ we obtain $t_{\delta_{1}}^{-1}=t_{x_{1}} t_{c_{3}} t_{c_{1}}^{-1} t_{c_{1}}^{-1} t_{e_{1}}^{-1} t_{f_{1}}^{-1}$ and $t_{\delta_{2}}^{-1}=t_{x_{2}} t_{c_{5}} t_{e_{1}}^{-1} t_{f_{1}}^{-1} t_{c_{7}}^{-1} t_{c_{7}}^{-1}$. Finally, substituting these into the order-three element above and simplifying the 


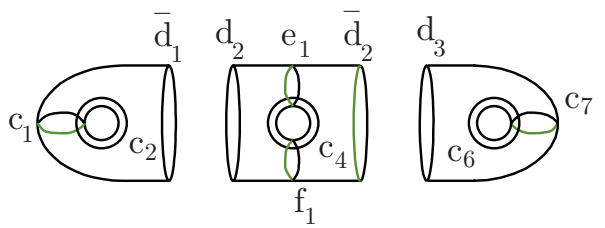

FiguRE 3

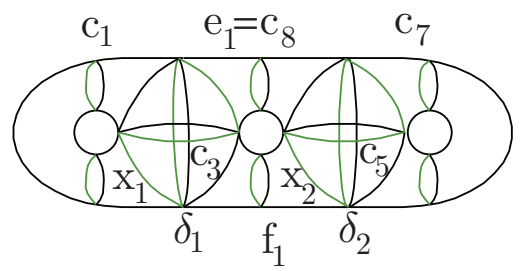

FiguRE 4

result using braid and commutativity relations (see $\S 6$ ) yields

$$
\begin{aligned}
& 1=\left(\left(t_{c_{1}} t_{c_{2}}\right)^{2}\left(t_{c_{4}} t_{e_{1}} t_{c_{4}} t_{f_{1}}\right)^{-1}\left(t_{c_{7}} t_{c_{6}}\right)^{2}\right)^{3} \\
& =\left(\left(t_{c_{1}} t_{c_{2}}\right)^{2} t_{\delta_{1}}^{-1}\left(t_{c_{4}} t_{e_{1}} t_{c_{4}} t_{f_{1}}\right)^{2} t_{\delta_{2}}^{-1}\left(t_{c_{7}} t_{c_{6}}\right)^{2}\right)^{3} \\
& =\left(\left(t_{c_{1}} t_{c_{2}}\right)^{2} t_{x_{1}} t_{c_{3}} t_{c_{1}}^{-1} t_{c_{1}}^{-1} t_{e_{1}}^{-1} t_{f_{1}}^{-1}\left(t_{c_{4}} t_{e_{1}} t_{c_{4}} t_{f_{1}}\right)^{2} t_{x_{2}} t_{c_{5}} t_{e_{1}}^{-1} t_{f_{1}}^{-1} t_{c_{7}}^{-1} t_{c_{7}}^{-1}\left(t_{c_{7}} t_{c_{6}}\right)^{2}\right)^{3} \\
& =\left(t_{c_{1}} \underline{t_{c_{2}} t_{c_{1}} t_{c_{2}}} t_{c_{1}}^{-1} t_{x_{1}} t_{c_{3}} t_{c_{1}}^{-1} \underline{t_{e_{1}}^{-1}} t_{f_{1}}^{-1} \underline{t_{c_{4}} t_{e_{1}} t_{c_{4}}} t_{f_{1}} \underline{t_{c_{4}} t_{e_{1}} t_{c_{4}}} t_{f_{1}}\right. \\
& \left.\cdot t_{x_{2}} t_{c_{5}} t_{e_{1}}^{-1} t_{f_{1}}^{-1} t_{c_{7}}^{-1} t_{c_{7}}^{-1} t_{c_{7}} t_{c_{6} t_{c_{7}} t_{c_{6}}}\right)^{3} \\
& =\left(t_{c_{1}} t_{c_{1}} t_{c_{2}} t_{c_{1}} t_{c_{1}}^{-1} t_{x_{1}} t_{c_{3}} t_{c_{1}}^{-1} t_{f_{1}}^{-1} t_{e_{1}}^{-1} t_{e_{1}} t_{c_{4}} t_{e_{1}} t_{f_{1}} t_{e_{1}} t_{c_{4}} t_{e_{1}} t_{f_{1}}\right. \\
& \left.\cdot t_{f_{1}}^{-1} t_{e_{1}}^{-1} t_{x_{2}} t_{c_{5}} t_{c_{7}}^{-1} t_{c_{7}}^{-1} t_{c_{7}} t_{c_{7}} t_{c_{6}} t_{c_{7}}\right)^{3} \\
& =\left(t_{c_{1}} t_{c_{1}} t_{c_{2}} t_{x_{1}} t_{c_{3}} t_{c_{1}}^{-1} t_{f_{1}}^{-1} t_{c_{4}} t_{f_{1}} t_{e_{1}} t_{e_{1}} t_{c_{4}} t_{x_{2}} t_{c_{5}} t_{c_{6}} t_{c_{7}}\right)^{3} \text {. }
\end{aligned}
$$

Pushing $t_{c_{1}}^{-1}$ to the right, as it commutes with every element to its right, eventually cancels a $t_{c_{1}}$ at the left end, and renaming $r:=t_{f_{1}}^{-1}\left(c_{4}\right)$ and $e_{1}=c_{8}$ results in the positive relator

$$
\varrho:=\left(c_{1} c_{2} x_{1} c_{3} r c_{8} c_{8} c_{4} x_{2} c_{5} c_{6} c_{7}\right)^{3} \in \mathcal{R} .
$$

Let $X_{3}$ be the Lefschetz fibration with monodromy $\varrho$. Let $X_{3,3}$ be the Lefschetz fibration whose monodromy is obtained from that of $X_{3}$ by three lantern substitutions, namely

$$
\varrho^{\prime}:=\left(\bar{y}_{1} x_{1} t v s_{2} c_{8} f_{1} c_{8} s_{2} \bar{x}_{2} r_{3}\right)^{3} .
$$

The following is how we show the equivalence of these two monodromies (see also Figure 5 and Figure 6). We will adapt the conjugation notation $r=f_{1}^{-1}\left(c_{4}\right)$ from now on. We apply elementary transformations and simultaneous conjugations to $\varrho$ 


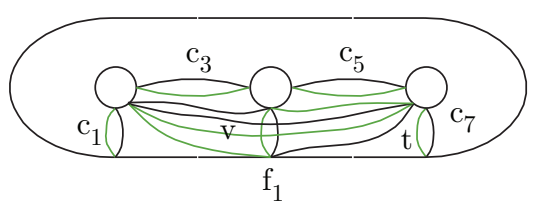

FiguRE 5

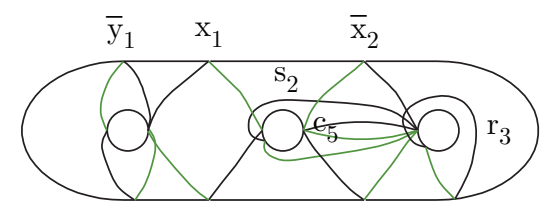

Figure 6

as follows:

$$
\begin{aligned}
\varrho & =\left(c_{1} c_{2} x_{1} c_{3} r c_{8} c_{8} c_{4} x_{2} c_{5} c_{6} c_{7}\right)^{3}=\left(c_{1} c_{2} x_{1} c_{3} \cdot f_{1}^{-1}\left(c_{4}\right) \cdot c_{8} c_{8} c_{4} x_{2} c_{5} c_{6} c_{7}\right)^{3} \\
& \sim f_{1}^{-1}\left(c_{1} c_{2} x_{1} c_{3} c_{4} c_{8} c_{8} \cdot f_{1}\left(c_{4}\right) \cdot x_{2} c_{5} c_{6} c_{7}\right)^{3} \sim\left(c_{1} c_{2} x_{1} c_{3} c_{4} c_{8} c_{8} \cdot f_{1}\left(c_{4}\right) \cdot x_{2} c_{5} c_{6} c_{7}\right)^{3} \\
& \sim\left(c_{1}\left(c_{2}\right) \cdot c_{1} x_{1} c_{3} c_{4} c_{8} c_{8} \cdot f_{1}\left(c_{4}\right) \cdot x_{2} c_{5} c_{6} c_{7}\right)^{3} \\
& \sim\left(c_{1}\left(c_{2}\right) \cdot x_{1} c_{3} c_{4} c_{8} c_{8} \cdot f_{1}\left(c_{4}\right) \cdot x_{2} c_{5} c_{6} c_{7} \cdot c_{1}\right)^{3} \\
& \sim\left(c_{1} \cdot c_{1}\left(c_{2}\right) \cdot x_{1} c_{3} c_{4} c_{8} c_{8} \cdot f_{1}\left(c_{4}\right) \cdot x_{2} c_{5} c_{6} c_{7}\right)^{3} \\
& \sim\left(c_{1} \cdot c_{1}\left(c_{2}\right) \cdot x_{1} c_{3} c_{4} c_{8} c_{8} \cdot c_{5} \cdot c_{5}^{-1} f_{1}\left(c_{4}\right) \cdot c_{5}^{-1}\left(x_{2}\right) \cdot c_{6} c_{7}\right)^{3} \\
& \sim\left(c_{1} \cdot c_{1}\left(c_{2}\right) \cdot x_{1} c_{3} c_{4} c_{5} c_{8} c_{8} \cdot f_{1} c_{5}^{-1}\left(c_{4}\right) \cdot \bar{x}_{2} \cdot c_{6} c_{7}\right)^{3} \quad\left(\bar{x}_{2}:={ }_{c_{5}^{-1}}\left(x_{2}\right)\right) \\
& \sim\left(c_{1} \cdot c_{1}\left(c_{2}\right) \cdot x_{1} c_{3} c_{5} \cdot c_{5}^{-1}\left(c_{4}\right) \cdot c_{8} c_{8} \cdot f_{1} c_{5}^{-1}\left(c_{4}\right) \cdot \bar{x}_{2} c_{7} \cdot c_{7}^{-1}\left(c_{6}\right)\right)^{3} \\
& \sim\left(c_{1} \cdot c_{1}\left(c_{2}\right) \cdot x_{1} c_{3} c_{5} \cdot s_{2} \cdot c_{8} c_{8} \cdot f_{1}\left(s_{2}\right) \cdot \bar{x}_{2} c_{7} \cdot r_{3}\right)^{3} \quad\left(s_{2}:={ }_{c_{5}^{-1}}\left(c_{4}\right), r_{3}:=c_{7}^{-1}\left(c_{6}\right)\right) \\
& \sim\left(c_{1} \cdot c_{1}\left(c_{2}\right) \cdot x_{1} c_{3} c_{5} c_{7} s_{2} c_{8} c_{8} \cdot f_{1}\left(s_{2}\right) \cdot \bar{x}_{2} r_{3}\right)^{3} \\
& \sim\left(c_{1}^{2}\left(c_{2}\right) \cdot c_{1} \cdot x_{1} c_{3} c_{5} c_{7} s_{2} c_{8} c_{8} \cdot f_{1}\left(s_{2}\right) \cdot \bar{x}_{2} r_{3}\right)^{3} \\
& \sim\left(\bar{y}_{1} x_{1} \cdot c_{1} c_{3} c_{5} c_{7} \cdot s_{2} c_{8} c_{8} \cdot f_{1}\left(s_{2}\right) \cdot \bar{x}_{2} r_{3}\right)^{3}=: \tau \quad\left(\bar{y}_{1}:=c_{1}^{2}\left(c_{2}\right)\right) . \\
&
\end{aligned}
$$

We apply elementary transformations to $\varrho^{\prime}$ as follows:

$$
\begin{aligned}
\varrho^{\prime} & =\left(\bar{y}_{1} x_{1} t v s_{2} c_{8} f_{1} c_{8} s_{2} \bar{x}_{2} r_{3}\right)^{3} \sim\left(\bar{y}_{1} x_{1} t v s_{2} c_{8} \cdot f_{1}\left(c_{8} s_{2} \bar{x}_{2} r_{3}\right) \cdot f_{1}\right)^{3} \\
& \sim\left(f_{1} \cdot \bar{y}_{1} x_{1} t v s_{2} c_{8} \cdot f_{1}\left(c_{8} s_{2} \bar{x}_{2} r_{3}\right)\right)^{3} \sim\left(f_{1}\left(\bar{y}_{1} x_{1}\right) \cdot f_{1} t v \cdot s_{2} c_{8} \cdot f_{1}\left(c_{8} s_{2} \bar{x}_{2} r_{3}\right)\right)^{3} \\
& =\left(\bar{y}_{1} x_{1} \cdot f_{1} t v \cdot s_{2} c_{8} c_{8} \cdot f_{1}\left(s_{2}\right) \cdot \bar{x}_{2} r_{3}\right)^{3}=: \tau^{\prime} .
\end{aligned}
$$

Thus $\tau^{\prime}$ is obtained by applying three times $L$-substitutions to $\tau$ by virtue of the lantern relation $f_{1} t v=c_{1} c_{3} c_{5} c_{7}$, and $M_{\varrho^{\prime}}=X_{3,3}$ turns out to be a rational blowdown of $M_{\varrho}=X_{3}$ along three copies of $C_{2}$ from Theorem 3.1

We set $\varsigma:=\left(c_{1} c_{2} c_{3} c_{4} c_{5} c_{6} c_{7}^{2} c_{6} c_{5} c_{4} c_{3} c_{2} c_{1}\right)^{2} \in \mathcal{R}$ and put $M_{1}:=M_{\varrho} \#_{F} M_{\varsigma}$ and $M_{2}:=M_{\varrho^{\prime}} \# F_{F} M_{\varsigma}$. Both of $M_{1}$ and $M_{2} \# 3 \overline{\mathbb{C P}}^{2}$ are simply-connected and have the Euler characteristic 56 and signature -36 . Hence Theorem 4.1 tells us that $M_{1}$, $M_{2} \# 3 \overline{\mathbb{C P}}^{2}$, and $\# 9 \mathbb{C P}^{2} \# 45 \overline{\mathbb{C P}}^{2}$ are homeomorphic but mutually non-diffeomorphic. 
We next exhibit an example of lantern substitution for genus 2 fibrations and pose a problem about it.

Example 5.3. Let $\varrho$ (resp. $\varrho^{\prime}$ ) be a positive relator of $\mathcal{M}_{2}$ given as follows (see Figure 7, Figure 8, Figure 9, and Figure 4 of [4):

$$
\begin{aligned}
\varrho & :=\left(c_{5} c_{4} c_{3} c_{2} c_{1}^{2} c_{2} c_{3} c_{4} c_{5}\right)^{2}, \\
\varrho^{\prime} & :={ }_{c_{3}}(\delta)_{c_{3} c_{4}^{-1}}(x) \cdot \bar{k} \bar{h} c_{5}{ }^{\cdot}{ }_{c_{1}^{-4}}\left(c_{2}\right)_{c_{1}^{-1}}\left(c_{2}\right)_{c_{2}^{-1} c_{3}}\left(c_{4}\right) \cdot k \cdot{ }_{c_{2}^{-1}}(h)_{c_{2}^{-1} c_{3}^{-1}}\left(c_{4}\right)_{c_{1}^{2}}\left(c_{2}\right) \cdot \bar{k} \bar{h} c_{5} c_{4} .
\end{aligned}
$$

Let $M_{\varrho}$ (resp. $M_{\varrho^{\prime}}$ ) be the corresponding Lefschetz fibration of genus 2 over $S^{2}$. It is well known that $M_{\varrho}$ is diffeomorphic to $\mathbb{C P}^{2} \# 13 \overline{\mathbb{C P}}^{2}$ (cf. [8]). $\varrho^{\prime}$ is obtained by applying elementary transformations and four times $L$-substitutions to $\varrho$ as follows:

$$
\begin{aligned}
& \varrho=\left(c_{5} c_{4} c_{3} c_{2} c_{1}^{2} c_{2} c_{3} c_{4} c_{5}\right)^{2} \\
& \sim c_{5} c_{4} c_{3} c_{2} c_{1} \cdot c_{1} c_{2} \cdot c_{3}\left(c_{4}\right) \cdot c_{3} c_{5} \cdot c_{5} c_{3} \cdot{ }_{c_{3}^{-1}}\left(c_{4}\right) \cdot c_{2} c_{1} \cdot c_{1} c_{2} c_{3} c_{4} c_{5} \\
& \stackrel{L}{\rightarrow} c_{5} c_{4} c_{3} c_{2} c_{1} \cdot c_{1} c_{2} \cdot c_{3}\left(c_{4}\right) \cdot c_{1} k h \cdot c_{3}^{-1}\left(c_{4}\right) \cdot c_{2} c_{1} \cdot c_{1} c_{2} c_{3} c_{4} c_{5} \\
& \sim c_{5} c_{4} c_{3} \cdot c_{1}^{2} \cdot c_{1}^{-2}\left(c_{2}\right) \cdot c_{2} \cdot c_{3}\left(c_{4}\right) \cdot c_{1} k h \cdot{ }_{c_{3}^{-1}}\left(c_{4}\right) \cdot c_{2} c_{1} \cdot c_{1} c_{2} c_{3} c_{4} c_{5} \\
& \sim c_{5}^{2} c_{1}^{2} \cdot c_{4} c_{3} \cdot{ }_{c_{1}^{-2}}\left(c_{2}\right) \cdot c_{2} \cdot c_{3}\left(c_{4}\right) \cdot c_{1} k h \cdot{ }_{c_{3}^{-1}}\left(c_{4}\right) \cdot c_{2} c_{1} \cdot c_{1} c_{2} c_{3} c_{4} \\
& \stackrel{L}{\rightarrow} c_{3} \delta x \cdot c_{4} c_{3} \cdot c_{1}^{-2}\left(c_{2}\right) \cdot c_{2} \cdot c_{3}\left(c_{4}\right) \cdot c_{1} k h \cdot{ }_{c_{3}^{-1}}\left(c_{4}\right) \cdot c_{2} c_{1} \cdot c_{1} c_{2} c_{3} c_{4} \\
& \sim \delta x c_{4} c_{3} \cdot{ }_{c_{1}^{-2}}\left(c_{2}\right) \cdot c_{2} \cdot c_{3}\left(c_{4}\right) \cdot c_{1} k h \cdot{ }_{c_{3}^{-1}}\left(c_{4}\right) \cdot c_{2} c_{1} \cdot c_{1} c_{2} c_{3} c_{4} c_{3} \\
& \sim \delta x c_{4} c_{3} \cdot c_{1}^{-2}\left(c_{2}\right) \cdot c_{2} \cdot c_{3}\left(c_{4}\right) \cdot c_{1} k h \cdot{ }_{c_{3}^{-1}}\left(c_{4}\right) \cdot c_{2} \cdot c_{1}^{2}\left(c_{2}\right) \cdot c_{1}^{2} c_{3}^{2} \cdot c_{3}^{-1}\left(c_{4}\right) \\
& \stackrel{L}{\rightarrow} \delta x c_{4} c_{3} \cdot c_{1}^{-2}\left(c_{2}\right) \cdot c_{2} \cdot c_{3}\left(c_{4}\right) \cdot c_{1} k h \cdot c_{3}^{-1}\left(c_{4}\right) \cdot c_{2} \cdot c_{1}^{2}\left(c_{2}\right) \cdot \bar{k} \bar{h} c_{5} \cdot c_{3}^{-1}\left(c_{4}\right) \\
& \sim c_{3}^{-1}\left(c_{4}\right) \cdot \delta x c_{4} c_{3} \cdot{ }_{c_{1}^{-2}}\left(c_{2}\right) \cdot c_{2} c_{1} \cdot{ }_{c_{3}}\left(c_{4}\right) \cdot k h \cdot{ }_{c_{3}^{-1}}\left(c_{4}\right) \cdot c_{2} \cdot c_{1}^{2}\left(c_{2}\right) \cdot \bar{k} \bar{h} c_{5} \\
& \sim c_{3}^{-1}\left(c_{4}\right) \cdot \delta x c_{4} c_{3} \cdot c_{1}^{-2}\left(c_{2}\right) \cdot c_{2} c_{1} c_{2} \cdot c_{2}^{-1} c_{3}\left(c_{4}\right) \cdot k \cdot c_{2}^{-1}(h)_{c_{2}^{-1}} c_{3}^{-1}\left(c_{4}\right)_{c_{1}^{2}}\left(c_{2}\right) \cdot \bar{k} \bar{h} c_{5} \\
& \sim c_{3}^{-1}\left(c_{4}\right) \cdot \delta x c_{4} c_{3} \cdot c_{1}^{-2}\left(c_{2}\right) \cdot c_{1} c_{2} c_{1} \cdot c_{2}^{-1} c_{3}\left(c_{4}\right) \cdot k \cdot c_{2}^{-1}(h) c_{2}^{-1} c_{3}^{-1}\left(c_{4}\right)_{c_{1}^{2}}\left(c_{2}\right) \cdot \bar{k} \bar{h} c_{5} \\
& \sim c_{3}^{-1}\left(c_{4}\right) \cdot \delta x c_{4} c_{3} c_{1}^{2} \cdot c_{1}^{-4}\left(c_{2}\right)_{c_{1}^{-1}}\left(c_{2}\right)_{c_{2}^{-1} c_{3}}\left(c_{4}\right) \cdot k \cdot c_{2}^{-1}(h) c_{2}^{-1} c_{3}^{-1}\left(c_{4}\right)_{c_{1}^{2}}\left(c_{2}\right) \cdot \bar{k} \bar{h} c_{5} \\
& \sim c_{3}^{-1} c_{4} c_{3}(\delta)_{c_{3}^{-1} c_{4} c_{3}}(x)_{c_{3}^{-1}}\left(c_{4}\right) \cdot c_{4} c_{3} c_{1}^{2} \cdot{ }_{c_{1}^{-4}}\left(c_{2}\right)_{c_{1}^{-1}}\left(c_{2}\right)_{c_{2}^{-1} c_{3}}\left(c_{4}\right) \cdot k \\
& \cdot c_{2}^{-1}(h) c_{2}^{-1} c_{3}^{-1}\left(c_{4}\right)_{c_{1}^{2}}\left(c_{2}\right) \cdot \bar{k} \bar{h} c_{5} \\
& \sim c_{3}^{-1} c_{4} c_{3}(\delta) c_{c_{3}^{-1} c_{4} c_{3}}(x) \cdot c_{4} c_{3}^{2} c_{1}^{2} \cdot{ }_{c_{1}^{-4}}\left(c_{2}\right)_{c_{1}^{-1}}\left(c_{2}\right)_{c_{2}^{-1} c_{3}}\left(c_{4}\right) \cdot k \\
& \cdot c_{2}^{-1}(h) c_{2}^{-1} c_{3}^{-1}\left(c_{4}\right)_{c_{1}^{2}}\left(c_{2}\right) \cdot \bar{k} \bar{h} c_{5} \\
& \sim c_{4} \cdot c_{3}(\delta)_{c_{3} c_{4}^{-1}}(x) \cdot c_{3}^{2} c_{1}^{2} \cdot c_{1}^{-4}\left(c_{2}\right)_{c_{1}^{-1}}\left(c_{2}\right)_{c_{2}^{-1} c_{3}}\left(c_{4}\right) \cdot k \cdot c_{2}^{-1}(h)_{c_{2}^{-1} c_{3}^{-1}}\left(c_{4}\right)_{c_{1}^{2}}\left(c_{2}\right) \cdot \bar{k} \bar{h} c_{5} \\
& \sim c_{3}(\delta)_{c_{3} c_{4}^{-1}}(x) \cdot c_{3}^{2} c_{1}^{2} \cdot{ }_{c_{1}^{-4}}\left(c_{2}\right)_{c_{1}^{-1}}\left(c_{2}\right)_{c_{2}^{-1} c_{3}}\left(c_{4}\right) \cdot k \cdot{ }_{c_{2}^{-1}}(h)_{c_{2}^{-1} c_{3}^{-1}}\left(c_{4}\right)_{c_{1}^{2}}\left(c_{2}\right) \cdot \bar{k} \bar{h} c_{5} c_{4} \\
& \stackrel{L}{\rightarrow} c_{3}(\delta)_{c_{3} c_{4}^{-1}}(x) \cdot \bar{k} \bar{h} c_{5} \cdot c_{1}^{-4}\left(c_{2}\right)_{c_{1}^{-1}}\left(c_{2}\right)_{c_{2}^{-1} c_{3}}\left(c_{4}\right) \cdot k \cdot c_{2}^{-1}(h)_{c_{2}^{-1} c_{3}^{-1}}\left(c_{4}\right)_{c_{1}^{2}}\left(c_{2}\right) \cdot \bar{k} \bar{h} c_{5} c_{4} \\
& =\varrho^{\prime} \text {, }
\end{aligned}
$$

where the symbol $\stackrel{L}{\rightarrow}$ stands for an $L$-substitution. Thus $M_{\varrho^{\prime}}$ turns out to be a four times rational blowdown of $M_{\varrho} \approx \mathbb{C P}^{2} \# 13 \overline{\mathbb{C P}}^{2}$ along copies of $C_{2}$ from Theorem 3.1

We set $\varsigma:=\varrho \in \mathcal{R}$ and put $M_{1}:=M_{\varrho} \# F_{F} M_{\varsigma}$ and $M_{2}:=M_{\varrho^{\prime}} \#_{F} M_{\varsigma}$. Both $M_{1}$ and $M_{2} \# 4 \overline{\mathbb{C P}}^{2}$ are simply-connected and have the Euler characteristic 36 and 


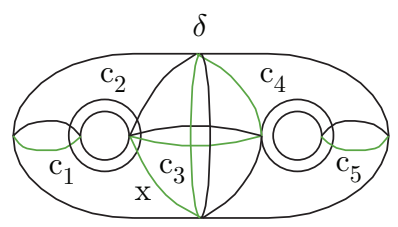

FiguRE 7
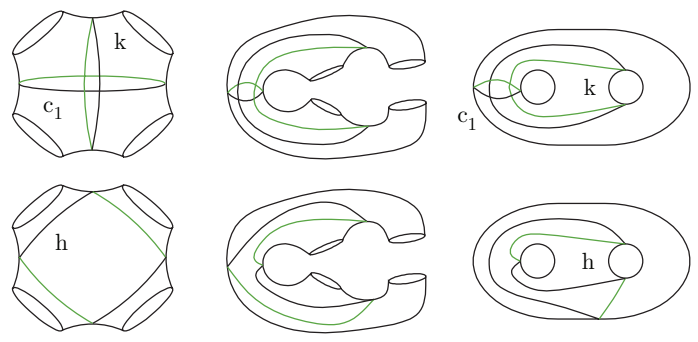

Figure 8
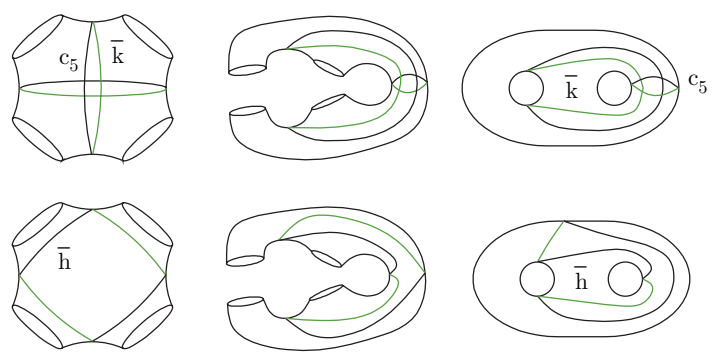

Figure 9

signature - 24. Hence Theorem 4.1 tells us that $M_{1}, M_{2} \# 4 \overline{\mathbb{C P}}^{2}$, and $\# 5 \mathbb{C P}^{2} \# 29 \overline{\mathbb{C P}}^{2}$ are homeomorphic but mutually non-diffeomorphic.

We denote the manifold $M_{\varrho^{\prime}}$ of Example 5.3 by $E$. Since $E$ is simply-connected and has the Euler characteristic 12 and signature $-8, E$ is homeomorphic to $E(1)=$ $\mathbb{C P}^{2} \# 9 \overline{\mathbb{C P}}^{2}$ from Freedman's classification theorem.

Problem 5.4. Does $E$ decompose into a non-trivial fiber sum of other Lefschetz fibrations? Is $E$ isomorphic to a fiber sum of two copies of Matsumoto's fibration?

Y. Sato told the first author that $E$ is not diffeomorphic to $E(1)$ because $E(1)$ does not admit a Lefschetz fibration of genus 2. If $E$ decomposes into a non-trivial fiber sum, then it is minimal by virtue of Usher's theorem [19. Matsumoto's fibration (Example B of [13]) is a Lefschetz fibration of genus 2 with 6 non-separating and 2 separating singular fibers, and total space diffeomorphic to $S^{2} \times T^{2} \# 4 \overline{\mathbb{C P}}^{2}$. It is easy to see that an appropriately twisted fiber sum of two copies of Matsumoto's fibration is homeomorphic but not diffeomorphic to $E(1)$. Another possible way to examine the manifold $E$ would be to compute the Seiberg-Witten invariants of $E$ by the rational blowdown formula of Fintushel and Stern [6]. 


\section{Other RELATIONS}

We finally observe effects of substitutions for other relations. Luo [1] improved Gervais' infinite presentation [7] of $\mathcal{M}_{g}$ to show that the set $\mathcal{R}$ of relators is normally generated by all commutativity, all braid, all 2-chain, and all lantern relators. We briefly review the definitions of the commutativity, braid and 2-chain relations.

Let $a, b$ be disjoint essential simple closed curves on $\Sigma_{g}$. The relation

$$
t_{a} t_{b}=t_{b} t_{a}
$$

in $\mathcal{M}_{g}$ is called a commutativity relation. A regular neighborhood $\Sigma$ of $a \cup b$ is the disjoint union of two annuli.

Let $a, b$ be simple closed curves on $\Sigma_{g}$ which intersect transversely at one point. The relation

$$
t_{a} t_{b} t_{a}=t_{b} t_{a} t_{b}
$$

in $\mathcal{M}_{g}$ is called a braid relation. A regular neighborhood $\Sigma$ of $a \cup b$ is a torus with one boundary component. Let $c$ be a simple closed curve parallel to the boundary of $\Sigma$. The relation

$$
\left(t_{a} t_{b}\right)^{6}=t_{c}
$$

in $\mathcal{M}_{g}$ is called a chain relation of length 2 , or a 2-chain relation for short.

Both sides of each relation above correspond to Lefschetz fibrations over $D^{2}$ with fiber $\Sigma$. It is not difficult to draw Kirby diagrams of those manifolds and find out what they are (cf. [8], Chapter 8). We actually obtain the manifolds in Table 1.

TABLE 1

\begin{tabular}{|c||c|c|c|}
\hline relation & manifold for LHS & manifold for RHS & common boundary \\
\hline commutativity & $D^{4} \amalg D^{4}$ & $D^{4} \amalg D^{4}$ & $S^{3} \amalg S^{3}$ \\
\hline braid & $X\left(S^{2},-2\right)$ & $X\left(S^{2},-2\right)$ & $\mathbb{R P}^{3}$ \\
\hline 2-chain & $M_{c}(2,3,6)$ & $X\left(T^{2},-1\right)$ & $\Sigma(2,3,6)$ \\
\hline lantern & $C_{2}$ & $B_{2}$ & $L(4,1)$ \\
\hline
\end{tabular}

The symbol $X(B, e)$ stands for the total space of a $D^{2}$-bundle over $B$ with Euler number $e$. The Milnor fiber $M_{c}(2,3,6)$ and the Brieskorn manifold $\Sigma(2,3,6)$ are defined by

$$
\begin{aligned}
M_{c}(2,3,6) & :=\left\{(x, y, z) \in \mathbb{C}^{3} \mid x^{2}+y^{3}+z^{6}=\varepsilon\right\} \cap D^{6}, \\
\Sigma(2,3,6) & :=\left\{(x, y, z) \in \mathbb{C}^{3} \mid x^{2}+y^{3}+z^{6}=0\right\} \cap S^{5}
\end{aligned}
$$

(see [8], Figure 8.13, for the Kirby diagram). Substitutions for commutativity and braid relations do not change the original manifold (cf. [20, Figure 34, and [3], Appendix A), whereas those for 2-chain and lantern relations do.

It might be interesting to extend Table 1 to that for various other relations, such as chain relations of length $n(\geq 3)$, the star relation, and Matsumoto's relations 12. No relation seems to be known to correspond to a rational blowing down process along $C_{p}$ for $p \geq 3$. 


\section{REFERENCES}

[1] J. Amorós, F. Bogomolov, L. Katzarkov, and T. Pantev, Symplectic Lefschetz fibrations with arbitrary fundamental groups, J. Diff. Geom. 54 (2000), 489-545. MR1823313(2002g:57051)

[2] M. Dehn, Die Gruppe der Abbildungsklassen, Acta Math. 69 (1938), 135-206. MR.1555438

[3] H. Endo, A generalization of Chakiris' fibrations, Groups of Diffeomorphisms, Advanced Studies in Pure Mathematics, 52, Mathematical Society of Japan, Tokyo, 2008, pp. 251-282.

[4] H. Endo and Y. Z. Gurtas, Positive Dehn twist expression for a $\mathbb{Z}_{3}$ action on $\Sigma_{g}$, preprint, arXiv:0808.0752.

[5] H. Endo and S. Nagami, Signature of relations in mapping class groups and non-holomorphic Lefschetz fibrations, Trans. Amer. Math. Soc. 357 (2005), 3179-3199. MR2135741 (2006g:57051)

[6] R. Fintushel and R. J. Stern, Rational blowdowns of 4-manifolds, J. Diff. Geom. 46 (1997), 181-235. MR1484044(98j:57047)

[7] S. Gervais, Presentation and central extensions of mapping class groups, Trans. Amer. Math. Soc. 348 (1996), 3097-3132. MR1327256 (96j:57016)

[8] R. E. Gompf and A. I. Stipsicz, 4-manifolds and Kirby calculus, Graduate Studies in Mathematics, 20, American Mathematical Society, Providence, RI, 1999. MR 1707327|(2000h:57038)

[9] D. Johnson, Homeomorphisms of a surface which act trivially on homology, Proc. Amer. Math. Soc. 75 (1979), 119-125. MR529227 (80h:57008)

[10] D. Kotschick, J. W. Morgan, and C. H. Taubes, Four-manifolds without symplectic structures but with nontrivial Seiberg-Witten invariants, Math. Res. Letters 2 (1995), 119-124. MR.1324695 (96i:57024)

[11] F. Luo, A presentation of mapping class groups, Math. Res. Lett. 4 (1997), 735-739. MR:1484704 (99b:57031)

[12] M. Matsumoto, A presentation of mapping class groups in terms of Artin groups and geometric monodromy of singularities, Math. Ann. 316 (2000), 401-418. MR1752777|(2001e:57002)

[13] Y. Matsumoto, Lefschetz fibrations of genus two - a topological approach, Proceedings of the 37th Taniguchi Symposium on "Topology and Teichmüller Spaces", World Scientific, Singapore, 1996, pp. 123-148. MR.1659687(2000h:14038)

[14] B. Ozbagci, Signatures of Lefschetz fibrations, Pacific J. Math. 202 (2002), 99-118. MR1883972 (2002k:57066)

[15] I. Smith, Lefschetz pencils and divisors in moduli space, Geometry \& Topology 5 (2001), 579-608. MR1833754(2002f:57056)

[16] C. H. Taubes, The Seiberg-Witten invariants and symplectic forms, Math. Res. Letters 1 (1994), 809-822. MR1306023 (95j:57039)

[17] C. H. Taubes, Counting pseudo-holomorphic submanifolds in dimension 4, J. Diff. Geom. 44 (1996), 818-893. MR1438194 (97k:58029)

[18] C. H. Taubes, The Seiberg-Witten and Gromov invariants, Math. Res. Letters 2 (1995), 221-238. MR1324704 (96a:57076)

[19] M. Usher, Minimality and symplectic sums, Int. Math. Res. Not. 2006, Art. ID 49857, 17 pp. MR2250015 (2007h:53139)

[20] K. Yasui, Elliptic surfaces without 1-handles, Journal of Topology 1 (2008), 857-878. MR2461858(2009i:57065)

Department of Mathematics, Graduate School of Science, Osaka University, ToyONAKA, OSAKA 560-0043, JAPAN

E-mail address: endo@math.sci.osaka-u.ac.jp

Department of Mathematics, DePauw University, 602 S. College Avenue, GreenCASTLE, Indiana 46135

E-mail address: yusufgurtas@depauw.edu

Current address: Department of Mathematics and Computer Science, Queensborough Community College-CUNY, 222-05 56th Avenue, Room S-245, Bayside, New York 11364

E-mail address: ygurtas@qcc.cuny.edu 